ELLEN REHM

\title{
Kleine Gaben für große Götter
}

Anlässlich des Rencontre Assyriologique Internationale in Harvard 1998 war es mir durch die außerordentliche Freundlichkeit von Timothy Kendall möglich, die wegen der Neugestaltung der Ausstellung nicht zugänglichen Bestände im Depot des Museums of Fine Arts in Boston zu besichtigen. Dabei entdeckte ich sechs silberne Votivbleche ${ }^{1}$ der Art, wie sie sich auch in Brüssel in den Musées Royaux d'Art et d'Histoire befinden, welche ich für einen Artikel im Gedenken an Peter Calmeyer bearbeiten durfte. ${ }^{2}$

Das große Problem aller Votivbleche ist, dass sie fast generell aus dem Kunsthandel stammen. Nur im Fall der Bleche aus dem sogenannten Oxus-Schatz ${ }^{3}$ kann man fast mit Sicherheit davon ausgehen, dass sie Weihgaben aus dem Tempel von Tacht-i Sangin waren. Bei den russischen Ausgrabungen wurden dort auch wenige weitere Artefakte dieser Fundgattung entdeckt. ${ }^{4}$ Bleche, die sich heute im Miho-Museum in Japan befinden, scheinen diese Gruppe zu ergänzen. 5 Zudem kam ein Goldblech in Nimrud zu Tage. ${ }^{6}$

1 Davon sind drei Bleche bereits als Neuerwerbung publiziert: T. Kendall, in: The Museum Year: 1988-1989. The One Hundred Nineteenth Annual Report of the Museum of Fine Art in Boston, 29. Für die Publikationserlaubnis der Bleche danke ich Dr. R. Freed.

2 Aufgrund des verspäteten Erscheinens dieser Publikation noch nicht zugänglich: E. Rehm, Votivbleche im 1. Jt. v. Chr. - Ausdruck unbekannter Kulte, in: Gedenkschrift für Peter Calmeyer, Altertumskunde des Vorderen Orients (im Druck).

3 O.M. Dalton, The Treasure of the Oxus with Other Examples of Early Oriental MetalWork, London, 1964, 19-26; Taf. XIV-XV.

4 I. R. Pitschikjan, Oxos-Schatz und Oxos-Tempel. Achämenidische Kunst in Mittelasien, Berlin 1992, $31 \mathrm{ff}$.

5 I. R. Pitschikjan (Pichikyan), Rebirth of the Oxus Treasure. Second Part of the Oxus Treasure from the Miho Collection, Ancient Civilizations from Scythia to Sibiria. An International Journal of Comparative Studies in History and Archeology 4 [1997] 312 (Es soll sich um 3000 Objekte handelt; Abbildungen sind bedauerlicherweise nicht vorhanden, aber eine Publikation ist vorgesehen).

${ }^{6}$ J. E. Curtis/K. R. Maxwell-Hyslop, The Gold Jewellery from Nimrud, Iraq 33 [1971] $101 \mathrm{ff}$. bes. 112; Taf. XXXI,1. Das oben zum Anhänger eingerollte Goldblech zeigt eine bartlose Gestalt mit erhobenen Händen. Es wurde im Schutt des Fort Salmanassar gefunden, Areal S. 
Bei meinen Untersuchungen der Votivbleche des 1. Jt. v. Chr. konnte als Ergebnis erschlossen werden, dass auf den urartäischen Blechen Götter ${ }^{7}$, auf den bekannten Blechen aus Mesopotamien und Iran hingegen im allgemeinen Beter dargestellt sind. Wie sehr nun diese Votivgaben ${ }^{8}$ dem Kult und der Anbetung verbunden sind, verdeutlichen zwei der Bostener Bleche.

Das Objekt Nr. 1 ( $A b b, 1, a)$ zeigt einen Beter, der vor einem größeren, auf einem Berg stehenden Gott die Hände bittend erhebt. Bei dieser Gottheit mag es sich vielleicht um Assur handeln', denn zum einen darf das Blech dem Stil der neuassyrischen Epoche angeschlossen werden, zum anderen kann der Reichsgott Assur mit dem Berg assoziiert werden. ${ }^{10}$ Auf dem anderen Blech (Nr. 4, $A b b .2, a)$ ist ebenfalls ein Beter mit erhobenen Armen dargestellt. Er befindet sich vor einem rechteckigen Altar, auf dem drei Spaten aufgestellt sind, die Symbole des babylonischen Gottes Marduk. Auf der anderen Seite des Altars steht auf einer Rampe, deren gewellte Form vielleicht Wasser wiedergeben soll, ein geflügeltes und gehörntes Mischwesen, das eventuell als ein Muschhusch ${ }^{11}$ oder als geflügelter Stier ${ }^{12}$ zu deuten ist. Die sich im oberen Bildfeld befindenden Astralsymbole Mond und Stern sind auch für vergleichbare neubabyloni-

7 E. Rehm, Kykladen und Alter Orient, Karlsruhe 1997, 195-197.

8 In den meisten Fällen sind die Bleche an den Ecken zwecks Befestigung durchbohrt (vgl. die urartäischen Bleche) oder gänzlich ohne irgendwelche Befestigungshilfen (vgl. die Oxus-Schatz Bleche). Zwei der hier besprochenen Bleche sind mit eingerollten Ösen versehen (Nr. 1. 4), was sie eher als Anhänger auszeichnet. Die bekannten Anhänger mit dieser Art von Ösen hingegen haben fast immer eine runde Form (vgl. Von Babylon bis Jerusalem. Die Welt der altorientalischen Königsstädte Bd. 1, Hrsg. W. Seipel, A. Wieczorek, Mannheim 1999, 138 Abb. 5; F. von Luschan, Die Kleinfunde von Sendschirli [Ausgrabungen in Sendschirli Bd. V] Berlin 1943, Taf. 46; R.-B. Wartke, Urartu. Das Reich am Ararat, Mainz 1993, 111 Abb. 53). Die rechteckige Form ohne verstärkte Ränder lässt m. E. eine Einordnung der Bleche Nr. 1 und Nr. 4 in die Gruppe der Votive zu, wenngleich die Verwandtschaft der Objekte nicht außer acht gelassen werden darf.

9 Vgl. für die Götterfigur die allerdings stärker bewaffnete Darstellung der Alabasterplatte aus Assur: G. R. Meyer, Altorientalische Denkmäler im Vorderasiatischen Museum zu Berlin, Leipzig 1965, Abb. 152.

10 D. O. Edzard, Assur, in: Wörterbuch der Mythologie Bd. 1, Hrsg. H. W. Haussig, Stuttgart 1965, 43f.; K. L. Tallqvist, Akkadische Götterepitheta, Hildesheim, New York 1974², 221. R. Mayer-Opificius machte mich darauf aufmerksam, dass auch Adad mit dem Berg in Verbindung gebracht werden kann, vgl. ihren Artikel: Das Relief des Schamaschresch-usur aus Babylon, in: Vom Alten Orient zum Alten Testament, Festschrift zum 85. Geburtstag von W. von Soden, AOAT 240, Hrsg. M. Dietrich/O. Loretz, NeukirchenVluyn 1995, 333-348.

11 Dieses Mischwesen wird sonst ungeflügelt dargestellt: U. Seidl, Die babylonischen Kudurru-Reliefs, OBO 87, Freiburg/Schweiz 1989, 187-192. Es geht aber auf das Tier des Ningischzida zurück, dessen Darstellungen es als geflügeltes Mischwesen zeigen. Vgl. W. Orthmann, Der Alte Orient, PKG 14, Berlin, 1975, 239 Fig. $44 \mathrm{f}$.

12 P. Albenda (A Mediterranean Seascape from Khorsabad, Assur 3/3 [1983] 25f.) weist dieses Wesen dem Wettergott Adad zu. 
sche Altarszenen aus der Glyptik belegt. ${ }^{13}$ Anders sieht es im iranischen Raum aus. Dass man dort für den achämenidischen Ahura Mazda keine Kultverehrung vor einer anthropomorphen Gottheit oder vor einem Symbol findet, verwundert nicht, denn diese sind in der persischen Kunst unbekannt. Nachdem eine Zeitlang von der Wissenschaft abgelehnt wurde, in der bemannten, geflügelten Sonnenscheibe den Hauptgott zu sehen, ist man inzwischen wieder zu der Meinung zurückgekehrt, hier eine Darstellung des Ahura Mazda vor sich zu haben. ${ }^{14}$ Der schwebende Gott begleitet zwar viele Szenen - wie u.a. Rollsiegeldarstellungen zeigen ${ }^{15}$ - oder schwebt in Eingängen von Persepolis ${ }^{16}$, ist aber nie Objekt der Verehrung auf einem Podest o. ä. ${ }^{17}$ Somit kann der anbetende Perser nur alleine auftreten ${ }^{18}$, hier als Bogenschütze mit Lanze (Nr. 5 , $A b b .2, b)$, gänzlich dem bekannten Typus auf den glasierten Ziegeln aus Susa ${ }^{19}$ entsprechend. Analog dazu sind auch die Darstellungen auf den Blechen Nr. 2 $(A b b .1, b)$ und Nr. 6. (Abb. 2,c) zu erklären, die Beter aus dem assyrisch-babylonischen. Kulturkreis in einer verkürzten Anbetungsszene - ohne Gottheit zeigen. ${ }^{20}$ Das Pferd (Nr. 3, Abb. 1,c), schon bei den Blechen aus dem OxusSchatz ${ }^{21}$ und aus dem Brüsseler Museum ${ }^{22}$ bekannt, könnte als Gabe für den Tempel gedeutet werden. Einige Bleche weisen Knickfalten auf, wie sie schon bei den Votiven aus dem Oxusgebiet bemerkt wurden. ${ }^{23}$ Sie scheinen in Bezug zur einer Darreichung in einem Tempel zu stehen.

Es ist unbekannt, wo und wie die Bleche entdeckt wurden. Sollten sie einen gemeinsamen Fundort haben, kann man - rein hypothetisch - nur von einem

13 Meist auf Stempelsiegeln werden die Symbole in zahlreichen Fällen einzeln wiedergegeben, zudem steht auf den Altären zusätzlich das Symbol des Nabu, der Griffel: L. Jakob-Rost, Die Stempelsiegel im Vorderasiatischen Museum, Mainz 1997, Nrn. 297-304. 313-322.

14 Zusammenfassend B. Jacobs, Das Chvarnah - Zum Stand der Forschung, MDOG 119 [1987] 215-243; vgl. auch E. Rehm, Der Schmuck der Achämeniden, AVO 2, Münster 1992, 268-274.

15 Vgl. z. B. E. F. Schmidt, Persepolis II (OIP 69) Chicago 1957, Taf. 3 (Siegel Nr. 2); Taf. 6 (Siegel Nr. 14); E. Porada, The Collection of the Pierpont Morgan Library. Corpus of Ancient Near Eastern Seals in North American Collections, Washington 1948, Taf. CXXV,831; D. J. Wiseman, Götter und Menschen im Rollsiegel Westasiens, London 1958, Nr. 106.

16 E. F. Schmidt, Persepolis I (OIP 86) Chicago 1953, Taf. 75-79.

17 In diesem Sinne ist wahrscheinlich auch die Bemerkung Herodots (I,131) zu verstehen: „Von den Sitten der Perser weiß ich folgendes: Es ist bei ihnen nicht üblich, Götterbilder, Tempel und Altäre zu errichten". (Übersetzung J. Feix, München 1963).

$18 \mathrm{Vgl}$ auch die zahlreichen Bleche aus dem Oxus-Schatz mit meist männlichen Figuren: Dalton, Anm. 3, 19-97 Nrn. 48-96.

19 The Royal City of Susa. Ancient Near Eastern Treasures in the Louvre, Hrsg. P. O. Harper, New York 1992, 226f. Nrn. 155-156.

20 Vgl. auch Rehm, Anm. 2., Abb. 3 (Inv.Nr. O.3436B, O.3436D, O.3436E, O.3436F, O.3436I, O.3436L, O.34360, O.3436P) und Curtis/Hyslop, Anm. 6.

21 Dalton, Anm. 3, 26 Nrn. 99-100; Taf. XI.

22 Rehm, Anm. 2, Abb. 4 (Inv.Nr. O.3436J, O.3436H, O.3436J, O.3436K).

23 R. D. Barnett, The Art of Bactria and the Treasure of the Oxus, IrAnt 8 [1968] 36f. 
Heiligtum mit unterschiedlichen Gottheiten ausgehen, das länger in Gebrauch war; beides ist nicht unüblich für den Vorderen Orient gewesen.

Obwohl diese Objekte aus dem Kunsthandel stammen und uns damit wichtige Informationen der Fundlagen verloren gegangen sind, zeigen diese Bleche dennoch, dass der Votivblechkult weiter verbreitet war, als bisher angenommen und bis auf Mesopotamien auszudehnen ist.

\section{Katalog}

1) Silber; H. ca. 5,7 cm; Inv.Nr. 198.150 (Abb. 1,a)

Das Blech hat eine rechteckig, an den Ecken abgerundete Form. An seiner Oberseite ist es nicht beschnitten, sondern wurde zu einer Zunge verlängert, die nach vorne hin zu einem Anhänger eingerollt wurde. Die getriebene Darstellung zeigt eine nach rechts gewandte menschliche Figur mit Bart. Auf dem Kopf thront eine oben verzierte Hörnertiara. In das meist von göttlichen Wesen getragene assyrische Schalgewand gekleidet, hat sie beide Arme erhoben. Bewaffnet ist sie mit zwei Bögen, die seitlich der Schultern zu sehen sind, und mit einem Schwert an der Taille. Sie steht auf einem durch Rautenmuster strukturierten, sich wellenartig nach oben verjüngenden Podest. Davor steht eine bärtige Gestalt in glattem, langem Mantel mit senkrecht verlaufendem Fransensaum, die die Hände anbetend erhoben hat.

\section{2) Silber; H. ca. 4,45 cm; Inv.Nr. 198.149 (Abb. 1,b)}

Die rechteckige Platte ist an den Seitenkanten ungleichmäßig eingezogen. Durch ihre oberen Ecken wurden große Löcher geschlagen. Auf der Fläche ist eine nach rechts gewandte, bärtige Figur angegeben, die mit einem knielangen, gegürteten Gewand bekleidet ist. Das Haupthaar scheint sorgfältig gekämmt und wird durch ein Band zusammengehalten, der Schopf bauscht sich im Nacken. Sorgfältig sind Augenbrauen, Augen und die Nase wiedergegeben. Auffällig ist der überproportional große Kopf, ein Phänomen, das bei den vorgestreckten Händen ebenfalls beobachtet wurde.

\section{3) Silber; H. ca. 2,5 cm; Inv.Nr. 1989.148 (Abb. 1,c)}

Unregelmäßig sind die Kanten des länglich rechteckigen Bleches gestaltet, auf dem die Darstellung eines gedrungenen Pferdes eingeritzt wurde. Es schreitet nach links und trägt oben auf dem Kopf einen Dekor, der zum Pferdegeschirr gehört.

4) Silber, H. ca. 4,8 cm; Inv.Nr. 1985.29 (Abb. 2,a)

Eine fast quadratische Form hat das Blech, an dessen Oberkante eine Zunge stehen blieb, die eingerollt einen Anhänger bildet. Die geritzte Darstellung besteht aus einer Gruppe: In der Mitte befindet sich ein durch Längs- und Schräglinien verzierter Altar, auf dem sich drei Spaten, Symbole des Gottes 
Marduk, befinden, von denen der mittlere die beiden äußeren etwas überragt. Links davon, wie auf einer wellenartigen Rampe stehend, zeigt sich ein geflügeltes Mischwesen mit zwei großen, leicht gebogenen Hörnern. Ritzungen charakterisieren seinen Körper, der Flügel ist federartig gestaltet. Es wird sich um den Muschhusch, das Attributivtier des Marduk handeln. Auf der anderen Seite des Altars steht eine bärtige Gestalt mit erhobenen Armen. Sie ist in ein Schal- oder Wickelgewand gekleidet, dessen Fransensäume durch Strichreihen angedeutet werden. Über der Szene schweben Mondsichel und Stern.

5) Silber, H. 4 cm; Inv.Nr. 1985.27 (Abb. 2,b)

In das schmale, länglich rechteckige Blech wurde die Darstellung einer nach rechts gewandten Person getrieben. Aufgrund guter Vergleiche aus Persepolis und Susa ist die Figur als persischer Gardist zu erkennen; gekleidet in ein persisches Faltengewand, bewaffnet mit Bogen, Köcher und Lanze.

6) Silber, H. 4,1 cm; Inv.Nr. 1985.28 (Abb. 2,c)

Abgerundet sind die Ecken des rechteckigen Bleches, in das die Zeichnung von zwei nach rechts schreitenden Personen mit erhobenen Armen getrieben wurde. Beide sind in ein langes, saumverziertes Gewand gekleidet, das in einem Fall eine Borte am Oberkörper, im anderen Fall eine am Rockteil zeigt. Die im Nacken gebauschten Haare ähneln sich, aber während der Anführer einen langen Bart trägt, ist die ihm folgende Figur glatt rasiert. 


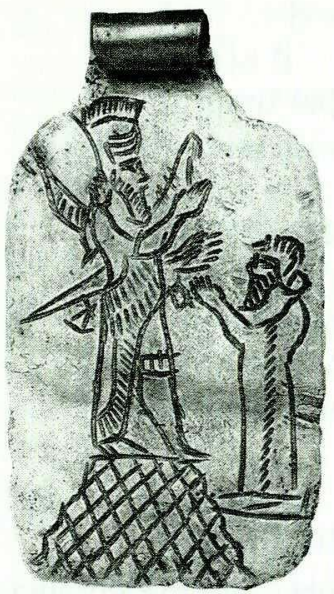

Abb. 1
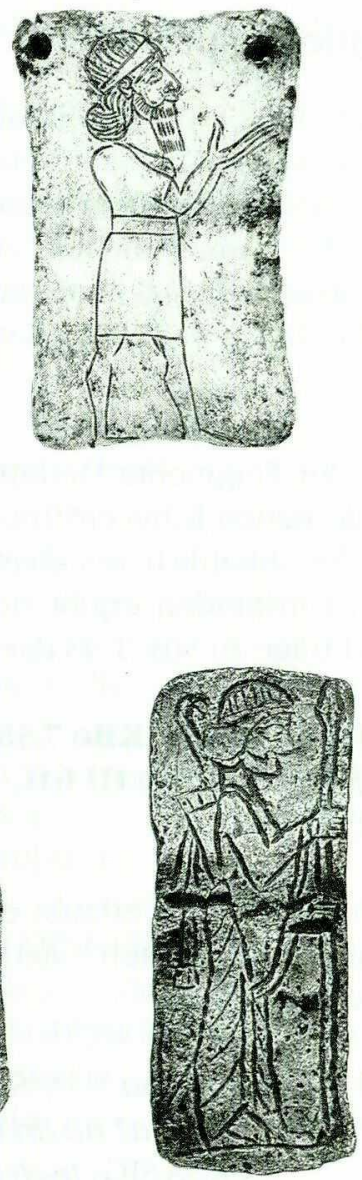
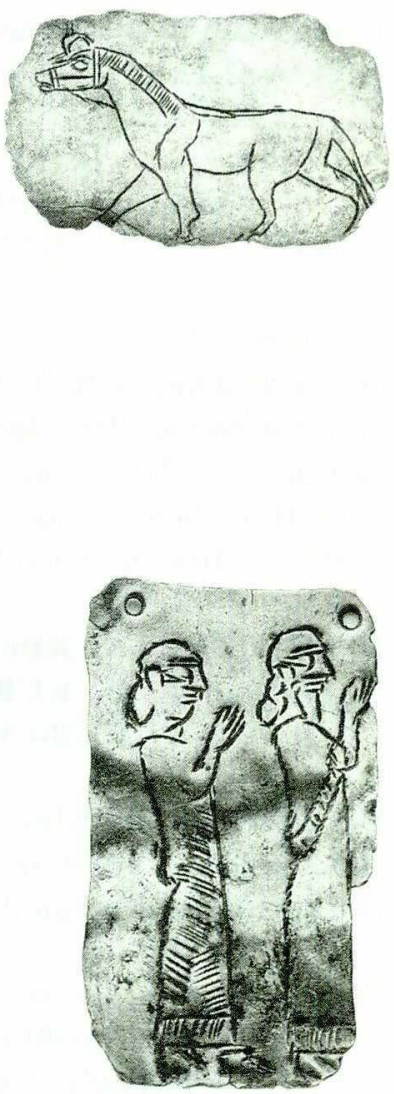

Abb. 2 